\title{
Barreiras para expansão do teletrabalho na pandemia COVID-19
}

Barriers to telework expansion in the COVID-19 pandemic

Barreras para la expansión del teletrabajo en la pandemia del COVID-19

Recebido: 03/06/2021 | Revisado: 09/06/2021 | Aceito: 10/06/2021 | Publicado: 26/06/2021

Rodrigo Rodrigues de Freitas
ORCID: https://orcid.org/0000-0002-8926-9588
Centro Federal de Educação Tecnológica Celso Suckow da Fonseca, Brasil
Universidade Federal do Rio de Janeiro, Brasil
E-mail: rodrigo.freitas@ cefet-rj.br
Thiago Ribeiro Sagawe
ORCID: https://orcid.org/0000-0002-2705-4506
E-mail: sagawethi @gmail.com
Aryane Cristine Dantas de Moura
Centro Federal de Educação Tecnológica Celso Suckow da Fonseca, Brasil
ORCID: https://orcid.org/0000-0002-5380-1747
E-mail: aryanecristine2@gmail.com
Fabrício Gomes Ribeiro
Centro Federal de Educação Tecnológica Celso Suckow da Fonseca, Brasil
ORCID: https://orcid.org/0000-0002-0436-1423
Centro Federal de Educaçãa Tecnológica Celso Suckow da Fonseca, Brasil
E-mail: fabricior673@gmail.com
Felipe do Carmo Amorim
ORCID: https://orcid.org/0000-0001-6658-0106
Eentro Federal de Educação Tecnológica Celso Suckow da Fonseca, Brasil
E-mail: felipe.amorim@cefet-rj.br

\section{Resumo}

A pandemia COVID-19, a crise do petróleo de 1973 e 1979, e o desemprego na Europa na década de 1980 tem em comum a quebra de paradigmas e mudanças comportamentais que exigiram transformações nas relações de trabalho. $\mathrm{O}$ avanço da tecnologia permitiu a aplicação do teletrabalho como alternativa para a redução do congestionamento e da poluição. Perceberam-se vantagens na melhoria da qualidade de vida e na redução de custos, porém mudanças comportamentais foram exigidas, de forma que o isolamento, trabalho autônomo e o senso de responsabilidade demandaram novas habilidades na sua aplicação. Foi realizada uma survey, a fim de comparar o resultado com o sistema sociotécnico em uma empresa de engenharia. Houve um consenso em reduzir entre um ou dois dias na semana, além de delegar mais responsabilidade aos funcionários, houve aceitação no aumento de carga horária para compensar a liberdade de escolha.

Palavras-chave: Teletrabalho; Trabalho em casa; Trabalho remoto; Sistema sociotécnico; COVID-19.

\begin{abstract}
The COVID-19 pandemic, the oil crisis of 1973 and 1979, and unemployment in Europe in the 1980s have in common the breaking of paradigms and behavioral changes that have required changes in labor relations. The advance of technology has enabled the application of telework as an alternative to reduce congestion and pollution. However, there were perceived advantages in improving the quality of life and reducing costs, but behavioral changes were required, so that isolation, self-employment, and a sense of responsibility demanded new skills in its application. A survey was carried out to compare the result with the sociotechnical system in an engineering company. There was a consensus to reduce by one or two days a week, in addition to delegating more responsibility to employees, there was an acceptance of increased workload to compensate for freedom of choice.
\end{abstract}

Keywords: Telework, Working from home, Remote work, Sociotechnical system, COVID-19.

\section{Resumen}

La pandemia del COVID-19, la crisis del petróleo de 1973 y 1979 y el desempleo en Europa en la década de 1980 tienen en común la ruptura de paradigmas y cambios de comportamiento que requerían transformaciones en las relaciones laborales. El avance de la tecnología permitió la aplicación del teletrabajo como alternativa para reducir la congestión y la contaminación. Se percibieron ventajas en mejorar la calidad de vida y reducir costos, pero se requirieron cambios de comportamiento, por lo que el aislamiento, el trabajo autónomo y el sentido de responsabilidad exigieron nuevas habilidades en su aplicación. Se realizó una survey para comparar el resultado con el sistema socio-técnico en una empresa de ingeniería. Hubo consenso para reducirlo entre uno o dos días a la semana, 
además de delegar más responsabilidad a los empleados, se aceptó un aumento en la carga de trabajo para compensar la libertad de elección.

Palabras clave: Teletrabajo, Trabajando en casa, Trabajo remoto, Sistema sociotécnico, COVID-19.

\section{Introdução}

Fatores externos são decisivos para a tomada de decisão das empresas, pois existe um trade-off entre a questão cultural e senso de responsabilidade (Vakilian \& Edrisi, 2020), quando afastam os funcionários dos escritórios outros mecanismos influenciam nas decisões como gênero, idade, experiência, nível de escolaridade, distância da residência até o local de trabalho entre outros (Vakilian \& Edrisi, 2020; Nakrošienė et al., 2019; O'Keefe et al., 2016; Caulfield, 2015). A pesquisa sugere que rompimentos históricos aceleram o processo de mudança estabelecidos por paradigmas e comportamentos sociais.

Na década de 1950, o desenvolvimento da telecomunicação criou um ambiente favorável ao deslocamento do trabalho tradicional em escritórios para outros locais (Baruch \& Yuen, 2000), duas hipóteses sugerem as primeiras tentativas de implantação do trabalho remoto. A primeira foi nas crises do petróleo na década de 1973 e 1979, em que a preocupação era o consumo de gasolina e os congestionamentos nas regiões metropolitanas do estado da Califórnia, nos Estados Unidos (Stiles, 2020), a segunda foi na década de 1980 com a necessidade de ampliar os negócios em locais diferentes da matriz com o rápido crescimento e impulso da tecnologia (Giovanis, 2017, Calderwood \& Freathy, 2014). Ambos os momentos possuem correlação com o avanço da tecnologia da comunicação, propiciando oportunidades de negócios fora do ambiente de escritório. Na Europa, o desenvolvimento de políticas de teletrabalho ocorreu na década de 1980 com alto nível de desemprego (Vakilian \& Edrisi, 2020; Caulfield, 2015), mesmo com o avanço tecnológico, barreiras culturais não permitiam o desenvolvimento na mesma velocidade (Stiles, 2020).

As vantagens do teletrabalho podem ser percebidas pela dimensão dos trabalhadores, empresas e meio ambiente. Os trabalhadores possuem a possibilidade de trabalhar em casa no caso de doença, economizar nas despesas de viagem, possibilidade em cuidar dos familiares (Nakrošienè et al., 2019). As empresas podem reduzir o custo operacional e o número de funcionários, proporcionando oportunidades para empreender em espaços menores (Saludin et al., 2020; Giovanis, 2017; O'Keefe et al., 2016; Silva-Send et al., 2013). Além disso, o meio ambiente é beneficiado com redução de gases poluentes, ruídos do tráfego e congestionamento, favorecendo a saúde pública (Giovanis, 2017).

Existe uma resistência dos gerentes quanto às mudanças para o trabalho em casa (Saludin et al., 2020), pois exigem maior disciplina, produtividade e qualidade na execução das atividades (Karia et al., 2003). O afastamento do funcionário no ambiente de trabalho reduz o senso de pertencimento e de encarreiramento, dificultando o acesso ao cliente (Vakilian \& Edrisi, 2020). Os funcionários debatem questões emocionais e estruturais relacionados a comer, beber e fumar em excesso (Henderson \& Mokhtarian, 1996), e a falta de estrutura tecnológica para ser realizado em casa. A dificuldade na implantação do trabalho remoto está mais direcionada aos fatores atitudinais do funcionário do que a questões demográficas, pois a localidade interfere sobre diferentes efeitos nas preferências das pessoas (Araújo et al., 2021; Okano et al., 2020; Mokhtarian \& Salomon, 1995).

A finalidade da pesquisa foi analisar os pressupostos do teletrabalho de acordo com a narrativa do sistema sociotécnico em período de choque social, pois a interação social, o desenvolvimento de equipes semiautônomas e delegação de atividades foram barreiras para aplicação. A segunda parte descreve a pesquisa de percepção antes e depois da pandemia COVID-19 com os trabalhadores. Na terceira e na quarta parte estão a metodologia e a comparação da pesquisa, com abordagem do sistema sociotécnico na equipe experimental. A conclusão e recomendações para futuras pesquisas na quinta parte. 


\subsection{Percepção antes e depois da Covid-19 na cidade do Rio de Janeiro}

A survey realizada propôs questionar a percepção em relação ao teletrabalho antes e depois da COVID-19 (Freitas et al., 2020). Aconteceu entre 05 e 31 de maio de 2020, e obteve 234 respostas com trabalhadores dos municípios da região metropolitana do estado do Rio de Janeiro. A região apresenta população de 12.3 milhões pessoas, e 55,72\% dos entrevistados residem na capital. Nessa região ocorre um deslocamento de 2 milhões de pessoas por dia, aumentando a população da capital em aproximadamente $30 \%$ por moradores de outros municípios (IETS, 2013).

Os resultados das Tabelas 1 e 2 destacam que entre os entrevistados, que não têm filhos e que têm filhos, 74,17\% e 71,08\% respectivamente, gostam do ambiente, dos colegas e da estrutura da empresa. Entre os que não têm filhos, 53,64\% prefeririam trabalhar em casa uma ou duas vezes na semana, os que têm filhos, 66,27\%. Os filhos e idade são fatores determinantes para aceitação, nas faixas etárias houve aumentos entre aqueles que prefeririam ficar pelo menos dois dias em casa., entre os adultos com idade de 18 a 29 anos, 54,13\%; 30 a 44 anos, 59,76\%; e 45 a 60 anos, 64,10\%. Em relação ao gênero as mulheres representam $65,55 \%$, e homens $50,43 \%$. As mulheres com filhos representam 82,05\%; sem filhos, $57,5 \%$. Os homens com filhos; $52,27 \%$, sem filhos, $49,29 \%$. Nos aspectos mencionados, as mulheres possuem maior aceitação para o teletrabalho. Fatores culturais, segurança, mobilidade e relações trabalhistas por regionalidade podem interferir na escolha. Pesquisa realizada por Nakrošienė et al. (2019), diz que não é verdade que os mais idosos e mulheres preferem o teletrabalho.

Tabela 1. Pesquisa de percepção do teletrabalho antes e depois da COVID-19.

\begin{tabular}{lcc}
\hline \multicolumn{1}{c}{ A sua função na empresa pode ser realizada em casa? } & Antes & Depois \\
\hline Sim, eu posso trabalhar em casa & 86 & 125 \\
\hline Parcialmente, mas preciso ir pelo menos um dia da semana na empresa & 25 & 27 \\
\hline Parcialmente, mas preciso ir pelo menos dois dias da semana na empresa & 17 & 12 \\
\hline Parcialmente, mas preciso ir pelo menos três dia da semana na empresa & 42 & 26 \\
\hline Não, pois é fundamental estar no ambiente da empresa & 64 & 44 \\
\hline \multicolumn{1}{c}{ Você se sentiria menos estressado se...? } & Antes & Depois \\
\hline Pelo menos um dia em casa, produziria a mesma coisa ou mais & 70 & 56 \\
\hline Pelo menos dois dias em casa, produziria a mesma coisa ou mais & 77 & 72 \\
\hline Pelo menos três dias em casa, produziria a mesma coisa ou mais & 24 & 28 \\
\hline Eu estiver todos os dias na empresa & 45 & 40 \\
\hline Todos os dias em casa, produziria a mesma coisa ou mais & 18 & 38 \\
\hline & Antes & Depois \\
\hline Pelo menos uma vez na semana se eu trabalhasse em casa & 59 & 47 \\
\hline Pelo menos duas vezes na semana se eu trabalhasse em casa & 77 & 75 \\
\hline Pelo menos três vez na semana se eu trabalhasse em casa & 33 & 37 \\
\hline Somente se eu trabalhasse em casa & 16 & 39 \\
\hline Gosto de ir todos os dias para o trabalho, me sinto melhor & 49 & 36 \\
\hline Você está disposto a trabalhar quantas horas em casa para obter mais tempo para & Antes & Depois \\
\hline saúde descanso, lazer ou entretenimento? & 104 & 110 \\
\hline Até completar a minha tarefa diária como se eu estivesse na empresa & 25 & 20 \\
\hline Menos que 8h & 66 & 66 \\
\hline Até 8h de trabalho & 27 & 24 \\
\hline Até 10h de trabalho & 12 & 14 \\
\hline Até 12h de trabalho & & \\
\hline
\end{tabular}

Fonte: Freitas et al. (2020).

A compensação pela liberdade de trabalhar em casa, pelo menos uma vez por semana, para cuidar da saúde, descansar e entretenimento, ainda que nesse dia se trabalhe mais que no expediente normal (Lister \& Harnish, 2019). As pessoas que 
participam de teletrabalho relataram uma melhora na qualidade de vida e diminuição no número de viagens realizadas, além de usar o tempo de viagem para trabalhar mais, aumentando a produtividade (Okano et al., 2020; O'Keefe et al., 2016). Percebe-se que $88,89 \%$ das pessoas possuem celular e computador próprios em casa, mesmo a pesquisa sendo realizada via formulário eletrônico, as respostas foram análogas por outros pesquisadores.

Tabela 2. Dados básicos dos entrevistados.

\begin{tabular}{|c|c|c|c|}
\hline \multicolumn{2}{|c|}{ Gênero } & \multicolumn{2}{|l|}{ Você tem meios de comunicação? } \\
\hline Feminino & 119 & Sim, telefone e computador próprios & 208 \\
\hline Masculino & 115 & Sim, somente o telefone & 11 \\
\hline \multirow[t]{2}{*}{ Prefiro não dizer } & 0 & Sim, somente o computador & 8 \\
\hline & & Sim, porém ambos são da empresa & 6 \\
\hline \multicolumn{2}{|c|}{$\begin{array}{l}\text { Pessoas que possuem filhos } \\
\end{array}$} & Não & 1 \\
\hline Tem filhos & 83 & & \\
\hline \multirow[t]{2}{*}{ Não tem filhos } & 151 & Faixa etária & \\
\hline & & 18 a 24 & 47 \\
\hline \multicolumn{2}{|c|}{ Setores da Economia em que trabalha } & 25 a 29 & 62 \\
\hline Indústria e Transformação & 65 & 30 a 34 & 39 \\
\hline Produto e Serviço & 167 & 35 a 39 & 22 \\
\hline \multirow[t]{2}{*}{ Agricultura e Extração } & 2 & 40 a 44 & 21 \\
\hline & & 45 a 49 & 12 \\
\hline \multicolumn{2}{|c|}{ Quanto anos tem de experiência laboral } & 50 a 54 & 15 \\
\hline Menos de 1 ano & 22 & 55 a 59 & 12 \\
\hline 1 a 3 & 36 & Acima de 60 & 4 \\
\hline 4 a 6 & 41 & & \\
\hline 7 a 9 & 23 & \multicolumn{2}{|c|}{ Você gosta do ambiente onde trabalha? } \\
\hline 10 a 15 & 36 & Sim, dos colegas e estrutura da empresa & 171 \\
\hline 16 a 20 & 23 & Sim, somente da estrutura & 10 \\
\hline 21 a 25 & 25 & Sim, dos colegas e da estrutura & 25 \\
\hline 26 a 30 & 11 & Sim, somente dos colegas & 22 \\
\hline 31 a 35 & 10 & Não & 5 \\
\hline Acima de 35 anos & 7 & Sim, somente do chefe & 1 \\
\hline
\end{tabular}

Fonte: Freitas et al. (2020).

\section{Metodologia}

O Sistema sociotécnico é uma abordagem relacionado a equipes semiautônomas com funções interdependentes em arranjos não-lineares entre as habilidades técnicas e sociais derivada dos sistemas complexos (Terra \& Passador, 2018; Trist \& Bamforth, 1951). A aplicação foi observada como oportunidade de modelos e estruturas de trabalhos que podem ser desenvolvidas a partir funções autônomas e gerenciadas a distância com a utilização da tecnologia de comunicação, que deve permitir o aprendizado contínuo, em que todos possam interagir em composição heterogênea e multidisciplinar (Mesquita et al., 2006). Existem dois subsistemas: técnico e social, o primeiro está relacionado às condições técnicas de instalação, equipamentos e ferramentas, o enfoque é a eficiência potencial da organização, o segundo está relacionado às relações sociais, psicológicas e fisiológicas para aqueles que executam a tarefa, o enfoque está em potencializar a eficiência (Trist \& Bamforth, 1951), as duas partes podem ser observadas na estrutura de trabalho com as funções da empresa para aperfeiçoar a capacidade de coordenação e controle pelos envolvidos na equipe entre o ambiente interno e externo da organização.

O termo sociotécnico foi associado ao grupo de trabalho (Emery \& Trist,1965), contudo, recentemente foi citado 
como um meio de mudanças de larga escala que transformam as funções da sociedade de forma mais autônoma por meio de tecnologias (Geels, 2010, 2018). A mudança estrutural, no caso do teletrabalho, é percebida com a diminuição da ênfase no social para aumento da ênfase na técnica, ou no fortalecimento nas relações entre as atividades técnicas e sociais (Latour, 2001). Desta forma, pode-se tratar como um conjunto de processos, regras e interações sociais para maior independência do trabalhador, onde o enfoque deixa de ser no controle da produtividade e passa a ser direcionado a resultados (Salerno, 2004). A avaliação intrínseca é observada de forma expandida para todos os funcionários, mantendo-se o controle da produtividade mesmo a distância (Garcia, 1980).

O objetivo é verificar as barreiras que impedem a aplicação do teletrabalho. A sistematização foi configurada pela perspectiva multinível (PMN) definida como multidisciplinar (Geels, 2010; Geels 2018; Stiles, 2020), em três níveis integrados e complementares: nichos, regimes e passagem sociotécnica que é conceitualizada como um processo de coevolução em dinâmica constante de aprendizado (Moors et al., 2004). Os nichos são caracterizados pelas experiências na formação do conhecimento tácito e associativo sobre as atividades práticas da equipe de trabalho, onde estão localizadas os trabalhadores, tecnologia, qualidade de vida e produtividade. O regime sociotécnico são as práticas, regras e cultura institucional que sofrem externalidade, que na pesquisa, a pandemia da COVID-19, acelerou o processo de mudança e aceitação. A passagem sociotécnica compõe os elementos do macronível como infraestrutura, política, ambiente demográfico, valores sociais e paradigmas. A Figura 1 apresenta a interdependências dos fatores identificado nas principais pesquisas relacionadas ao teletrabalho. O processo apoiou-se nos sete princípios básicos de para a abordagem sociotécnica: unidade básica do trabalho, grupo de trabalho, autorregulação, variedade de funções, autonomia e liberdade de ação, complementaridade das partes, diversidade (Trist, 1981).

Figura 1. Esquema metodológico do sistema sociotécnico.

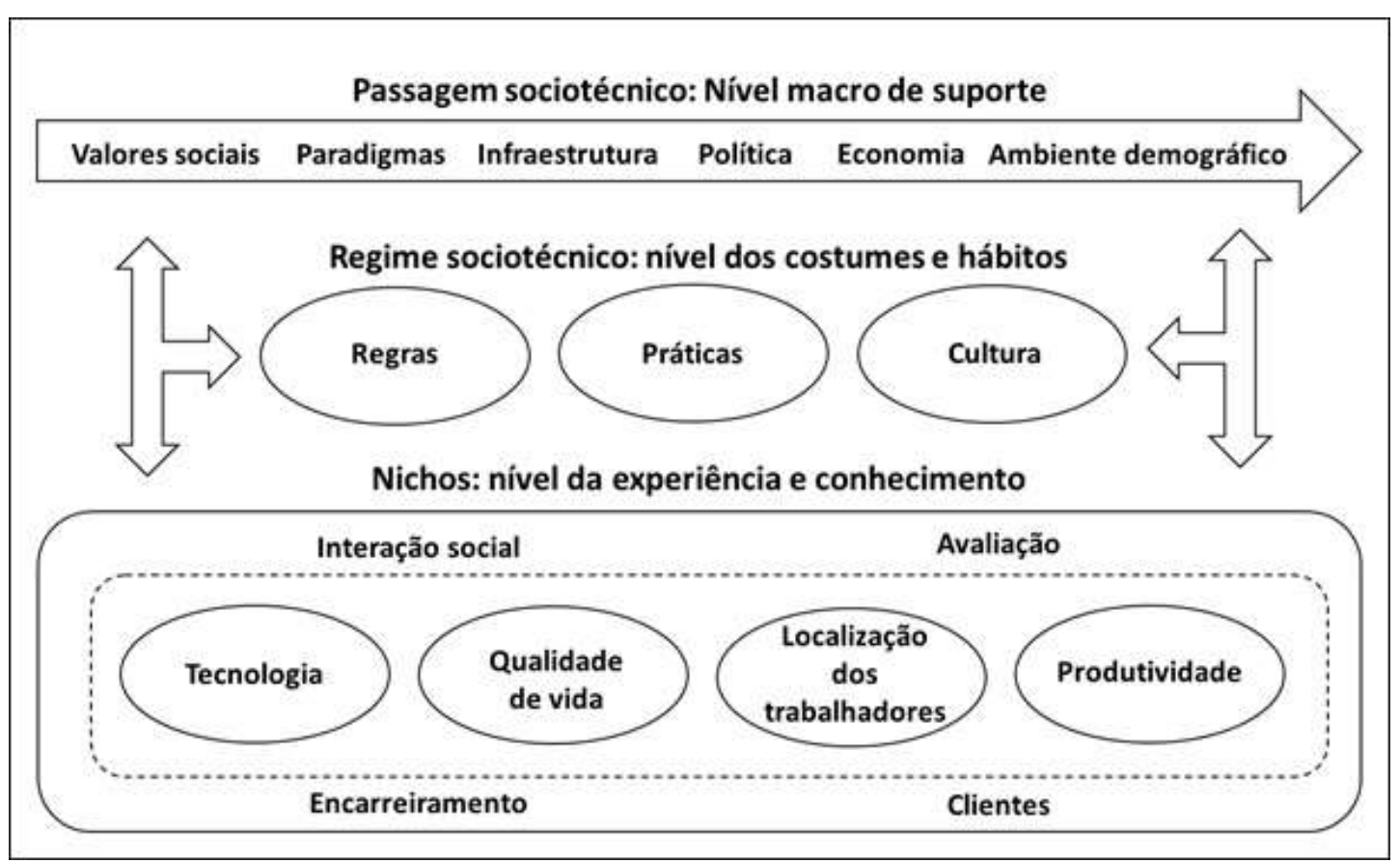

Fonte: Autores.

\section{Aplicação, Discursão e Resultados}

A execução foi observada em uma empresa de venda, manutenção e instalação de equipamentos de engenharia na cidade do Rio de Janeiro. A equipe experimental foi formada por seis mulheres (duas na faixa de 30 anos e quatro acima de 40 
anos), sete homens (todos acima de 40 anos). A experimentação começou em 16 de março, existia uma ideia para reduzir o número de dias em regime presencial na empresa, porém sem um plano estruturado. Em apenas uma reunião, a equipe foi forçada a admitir o teletrabalho como um benefício. Os funcionários aceitaram a redução salarial de $20 \%$, assim como a retirada do auxílio-transporte, o cenário de quarentena provocou uma aceleração nas condições de adesão do teletrabalho (Belzunegui-Eraso \& Erro-Garcés, 2020).

O enfoque dos gerentes foram os resultados (Salerno, 2004), pois como os funcionários estavam trabalhando em casa, aumentou-se a autonomia no período de dois meses, delegando autoridade e responsabilidade, os gerentes passaram a controlar a fronteira do processo como fechamentos de contratos, mas não o processo de negociação, onde todos os envolvidos participavam distribuindo o peso das decisões. A aceitação foi alta, contudo, foram observados problemas estruturais como mesas e cadeiras não adequadas, distração por dividir o mesmo espaço com outros membros da família e tempo em isolamento. A Tabela 3, descreve a comparação do comportamento dos trabalhadores, o antes e depois dos entrevistados na pesquisa.

Tabela 3. Comparação comportamental da equipe de trabalho com os entrevistados.

\begin{tabular}{|c|c|c|c|}
\hline \multirow[t]{2}{*}{ Percepção } & \multirow[t]{2}{*}{ Equipe de trabalho } & \multicolumn{2}{|c|}{$\begin{array}{c}\begin{array}{c}\text { Concordância dos } \\
\text { entrevistados }\end{array} \\
\end{array}$} \\
\hline & & Antes & Depois \\
\hline $\begin{array}{l}\text { Função que realiza na empresa } \\
\text { pode ser realizada em casa }\end{array}$ & $\begin{array}{l}\text { Todos os funcionários da empresa se adaptaram em } \\
\text { trabalhar em casa, contudo, aceitariam trabalhar alguns } \\
\text { dias em casa, mas não todos }\end{array}$ & $72,65 \%$ & $81,20 \%$ \\
\hline $\begin{array}{l}\text { Melhoraria a produtividade se } \\
\text { trabalhasse alguns dias em casa }\end{array}$ & $\begin{array}{l}\text { Os funcionários relataram que a produtividade está } \\
\text { como antes, porém não conseguem controlar o horário } \\
\text { de trabalho, alguns dias trabalham } 12 \mathrm{~h} \text {, outros dias } \\
\text { menos do que } 8 \mathrm{~h}\end{array}$ & $80,77 \%$ & $82,91 \%$ \\
\hline $\begin{array}{l}\text { Se sentiria menos estressado, se } \\
\text { trabalhasse pelos menos um dia da } \\
\text { semana em casa }\end{array}$ & $\begin{array}{l}\text { Os funcionários alegam que aumentou a qualidade de } \\
\text { vida, contudo o trabalho em equipe e o contato na } \\
\text { empresa é importante para o desenvolvimento e } \\
\text { crescimento profissional }\end{array}$ & $79,06 \%$ & $84,62 \%$ \\
\hline $\begin{array}{l}\text { Disposição para trabalhar mais em } \\
\text { casa para obter maior liberdade em } \\
\text { relação a horários }\end{array}$ & $\begin{array}{l}\text { Todos alegaram que a liberdade na escolha do horário } \\
\text { de trabalho é um benefício, pois podem planejar melhor } \\
\text { as atividades de entretenimento, saúde e descanso }\end{array}$ & $89,32 \%$ & $91,45 \%$ \\
\hline
\end{tabular}

Fonte: Autores.

Cada membro da equipe de trabalho possuía múltiplas responsabilidades, pois as variáveis do nicho foram mais perceptíveis para moldar o comportamento (Adamides, 2019), sendo influenciada pela interação social, avaliação, encarreiramento e clientes. O regime sociotécnico foi percebido no grau de responsabilidade de cada membro, pois optou-se por formar uma equipe composta por profissionais que possuem habilidade para o trabalho autônomo, que mesmo a distância, seguiram os padrões estabelecidos pela empresa. As variáveis do nível macro suportaram a passagem atitudinal de cada membro da equipe, entretanto foram observados problemas na estrutura e nos valores sociais.

A tecnologia permitiu que o grupo trabalhasse a distância, tornando a equipe interdependente das decisões finais que eram tomadas por todos e assessorada pelos gerentes, onde a avaliação era realizada por todos os membros. A variedade de funções e a transparência facilitou o desenvolvimento do grupo, o tornando mais experientes (Terra \& Passador, 2018; Geels, 2018), de forma que as decisões de cada membro eram pertinentes ao senso de responsabilidade. As vantagens foram a redução de custo operacional, espaço e pessoal para a empresa (Vakilian \& Edrisi, 2020; Saludin et al., 2020; O'Keefe et al., 2016; Silva-Send et al., 2013). Os funcionários também relataram que houve aumento das despesas em casa, contudo com menos deslocamento e liberdade para escolher o horário de trabalho, a diferença foi compensada. $\mathrm{O}$ trabalho remoto entre uma ou duas vezes na semana, para os tipos de trabalho que permitem essa aplicação, possivelmente será expandido para as demais 
funções da empresa (Lister \& Harnish, 2019).

\section{Conclusão e Sugestões para Pesquisas Futuras}

O teletrabalho exige mudanças comportamentais e quebra de paradigmas estabelecidos pelas empresas e trabalhadores. A tecnologia funciona como um vetor de mudança, contudo somente a sua aplicação, não permitiu a aceitação para o teletrabalho. Para identificar as principais variáveis de mudança foi realizado uma survey de percepção dos trabalhadores antes e depois da COVID-19 e comparada com resultados da abordagem do sistema sociotécnico em uma empresa de engenharia. Existe uma aceitação na aplicação do teletrabalho, contudo os trabalhadores preferiram trabalhar no máximo um ou dois dias em casa, aceitando o aumento de carga horária para obterem mais liberdade de escolha de horário.

Trabalhadores com filhos e idade mais avançada são fatores que contribuem para a decisão. As mulheres possuem maior aceitação para o teletrabalho, porém não foram abordados fatores culturais, segurança, mobilidade e relações trabalhistas. Acredita-se que a regionalidade pode interferir na escolha da adoção do trabalho remoto.

Os resultados apontam para futuras contribuições no desenvolvimento de autenticação remota, como a tecnologia blockchain e o aumento da segurança cibernética. Com a expansão do conceito, deve-se discutir leis trabalhistas para o trabalho remoto, pois funcionários podem ser registrados em países com a legislação diferente do local de atuação.

\section{Referências}

Adamides. E. D. (2019). Activity-based analysis of sociotechnical change. Syst Res Behav Sci. 1-12. http://dx.doi.org/10.1002/sres.2616.

Araujo, I. C., Luquini, I. M. A. M., Castro, M. C. D., Maia, P. L. O., \& Esteves, D. P. (2021). Teleworking: considerations and implications of employment relationships for workers' health. Research, Society and Development 10, n. 1, p. e50010111995. https://doi.org/10.33448/rsd-v10i1.11995.

Baruch, Y., \& Yuen, Y. K. J. (2000). Inclination to opt for teleworking: A comparative analysis of United Kingdom versus Hong Kong employees. International Journal of Manpower 21(7), 521-539. https://doi.org/ 10.1108/01437720010378980.

Belzunegui-Eraso, A., \& Erro-Garcés, A. (2020). Teleworking in the Context of the Covid-19 Crisis. Sustainability 12(9), 3662. https://doi.org/10.3390/ su12093662.

Calderwood, E., \& Freathy, P. (2014). Consumer mobility in the Scottish isles: The impact of internet adoption upon retail travel patterns. Transportation Research Part A: Policy and Practice 59, 192-203. https://doi.org/10.1016/j.tra.2013.11.012.

Caulfield, B. (2015). Does it pay to work from home? Examining the factors influencing working from home in the Greater Dublin Area. Case Studies on Transport Policy 3, 206-214. http://dx.doi.org/10.1016/ j.cstp.2015.04.004.

Emery, F. E., \& Trist, E.L., (1965). The causal textures of organizational environments. Human Relations 18, 21-32. https://doi.org/10.1177/ 001872676501800103.

Freitas, R. R., Sagawe, T. R., Moura, A. C. D., Ribeiro, F., \& Amorim, F. C. (2020). COVID-19 e o Teletrabalho: pesquisa de percepção de antes e durante a pandemia na região metropolitana do Rio de Janeiro. CEFET-RJ Campus Itaguaí. https://doi.org/10.5281/zenodo.3941215.

Garcia, R. M. (1980). Abordagem Sócio-técnica: Uma rápida avaliação. Rev. Adm. Emp. 20 (3), 71-77. https://doi.org/10.1590/S0034-75901980000300006.

Geels, F. W. (2010). Ontologies, socio-technical transitions (to sustainability), and the multi-level perspective. Research Policy 39(4), 495-510. https://doi.org/10.1016/j.respol.2010.01.022.

Geels, F. W. (2018). Disruption and low-carbon system transformation: Progress and new challenges in socio-technical transitions research and the multi-level perspective. Energy Research \& Social Science 37, 224-231. https://doi.org/10.1016/j. erss.2017.10.010.

Giovanis, E. (2017). The relationship between teleworking, traffic and air pollution. Atmospheric Pollution Research. http://dx.doi.org/ 10.1016/j.apr.2017.06.004.

Henderson, D., \& Mokhtarian, P. (1996). Impacts of center-based telecommuting on travel and emissions: analysis of the puget sound demonstration project. Transp. Res. D 1 (1), 29-45. https://doi.org/10.1016/ S1361-9209(96)00009-0.

IETS - Instituto de Estudos do Trabalho e Sociedade (2013). Mobilidade urbana e mercado de trabalho na região metropolitana do Rio de Janeiro: Estudo estratégico 06.

Karia, N; Zainuddin, Y., \& Asaari, M. H. (2003). Perception of Human Resources Managers on Telecommuting Concept Implementation in Malaysian Firms. Gajah Mada International Journal of Business 5 (1), 97-107. https://doi.org/10.22146/gamaijb.5395.

Latour, B. (2001). A esperança de pandora: ensaios sobre a realidade dos estudos científicos. EDUSC. 
Lister, K., \& Harnish, T. (2019). Telework and its effects in the United States. Chapters, in: Jon C. Messenger (ed.), Telework in the 21 st Century, chapter 3 , pages 128-170, Edward Elgar Publishing.

Mesquita, R. A., Sakahara, R., Nakano, D. N., \& Santos, C. (2006). Análise da implantação da organização do trabalho em grupos em empresa do setor químico tendo como referência o modelo sociotécnico. In: XXVI ENEGEP. Brasil.

Mokhtarian, P., \& Salomon, I. (1997). Modeling the desire to telecommute: the importance of attitudinal factors in behavioral models. Transp. Res. A 31 (1).35-50. https://doi.org/10.1016/S0965-8564(96)00010-9.

Moors, E; Rip, A., \& Wiskerke, J. S. C. (2004). The Dinamics of Innovation: A Multilevel Coevolucionary Pespective. In: Seeds of transition: essays on novelty production, niches and regimes in agriculture. Wageningen: Royal Van Gorcum, 31-56.

Nakrošienè, A., Bučiūnienè, I., \& Goštautaitė, B. (2019). Working from home: characteristics and outcomes of telework. International Journal of Manpower, 0143-7720. https://doi.org/10.1108/IJM-07-2017-0172.

Okano, M. T.; Santos, H. de C. L. dos; Honorato, W. J.; Viana, A. M.; \& Ursini, E. L. (2020). Impacts of the Covid-19 pandemic on large companies: evaluation of changes in technology infrastructure for telework under the optics of theories of dynamic capacity and adaptive structure. Research, Society and Development 9, n. 9, p. e756997852. https://doi.org/ 10.33448/rsd-v9i9.7852.

O'Keefe, P., Caulfield, B., Brazil, W., \& White, P. (2016). The impacts of telecommuting in Dublin, Research in Transportation Economics. http://dx.doi.org/10.1016/j.retrec.2016.06.010.

Salerno, M.S. (2004). Da rotinização à flexibilização: ensaio sobre o pensamento crítico brasileiro de organização do trabalho. Revista Gestão e Produção 11 (1), 21-32.

Saludin, N.A., Karia, N., \& Hassan, H. (2020). Working from Home (WFH): Is Malaysia Ready for Digital Society?. Entrepreneurship Vision 2020: Innovation, Development Sustainability, and Economic Growth.

Silva-Send, N., Anders, S., \& Narwold, A. (2013). Cost effectiveness comparison of certain transportation measures to mitigate greenhouse gas emissions in San Diego County, California. Energy Policy 62, 1428-1433. http://dx.doi.org/10.1016/j.enpol.2013.07.059.

Stiles, J. (2020). Strategic niche management in transition pathways: Telework advocacy as groundwork for an incremental transformation. Environmental Innovation and Societal Transitions 34, 139-150. https://doi.org/10.1016/j.eist.2019.12.001.

Terra, L. A. A., \& Passador, J. L. (2018). The nature of social organization of production: From firms to complex dynamics. Syst. Res. Behav. Sci. 1-18. https://doi.org/10.1002/sres.2567.

Trist, E. L. (1981). The evolution of socio-technical system. Toronto, Ontario: Ministry of Labor/Ontario Quality of Working Life Center. Issues in the quality of working life: a serie of occasional papers, $\mathrm{n} 2$.

Trist, E. L., \& Bamforth, K. W. (1951). Some social and psychological consequences of the longwall method of coal-getting: An examination of the psychological situation and defences of a work group in relation to the social structure and technological content of the work system. Human Relations 4 (1), 3-38. https://doi.org/10.1177/001872675100400101.

Vakilian. R., \& Edrisi. A. (2020). Modeling Factors Affecting the Choice of Telework and Its Impact on Demand in Transportation Networks. Civil and Environmental Engineering. https://doi.org/ 10.2478/cee-2020-0003. 